\title{
LATERAL OROMANDIBULAR DEFECT: WHEN IS IT APPROPRIATE TO USE A BRIDGING RECONSTRUCTION PLATE COMBINED WITH A SOFT TISSUE REVASCULARIZED FLAP?
}

\author{
Douglas B. Chepeha, MD, MSPH, ${ }^{1}$ Theodoros N. Teknos, MD, ${ }^{1}$ Kevin Fung, MD, ${ }^{2}$ \\ Josef Shargorodsky, MD, ${ }^{1}$ Assuntina G. Sacco, BS, ${ }^{1}$ Brian Nussenbaum, MD, ${ }^{3}$ \\ Lamont Jones, MD, ${ }^{1}$ Avraham Eisbruch, MD, ${ }^{4}$ Carol R. Bradford, MD, MS, ${ }^{1}$ \\ Mark E. Prince, MD, ${ }^{1}$ Jeffrey S. Moyer, MD, ${ }^{1}$ Julia S. Lee, MPH, MS, ${ }^{5}$ Gregory T. Wolf, MD ${ }^{1}$ \\ ${ }^{1}$ Department Otolaryngology-Head and Neck Surgery, University of Michigan, Ann Arbor, \\ Michigan 48109-0312. E-mail: dchepeha@umich.edu \\ 2 Department Otolaryngology-Head and Neck Oncology and Reconstructive Surgery, \\ University of Western Ontario, London, Ontario, Canada \\ ${ }^{3}$ Department Otolaryngology-Head and Neck Surgical Oncology, Washington University, \\ St. Louis, Missouri \\ ${ }^{4}$ Department of Radiation Oncology, University of Michigan, Ann Arbor, Michigan \\ ${ }^{5}$ Cancer Center-Biostatistics, University of Michigan, Ann Arbor, Michigan
}

Accepted 14 September 2007

Published online 19 February 2008 in Wiley InterScience (www.interscience.wiley.com). DOI: 10.1002/hed.20776

\begin{abstract}
Background. A quasi-experimental retrospective study was undertaken to evaluate a new concept of free tissue volume restoration combined with bridging reconstruction plate (compartment approach) to reduce plate-related complication rates.

Methods. We evaluated 40 patients with large lateral mandible defects and associated complex soft tissue defects reconstructed with a revascularized soft tissue flap and titanium hollow screw reconstruction plates. A case-control comparison was performed based on reconstruction type: restoration of soft tissue defect (conventional approach - group 1) versus over-reconstruction of soft tissue defect (compartment approach—group 2).

Results. Plate exposure rate was 6 of $16(38 \%)$ in group 1 versus 2 of $24(8 \%)$ in group 2, and the difference was statistically significant $(p=.04)$. The mean time to exposure was 10 months. Plate fracture rate was 6 of $23(26.1 \%)$ in dentulous patients versus 1 of 17 (5.9\%) in edentulous patients. Gastros-
\end{abstract}

Correspondence to: D. B. Chepeha

Dr. Chepeha has reported a financial interest/relationship with KLS Martin. ๑ 2008 Wiley Periodicals, Inc. tomy tube dependence was 6 of $16(38 \%)$ in group 1 versus 6 of $24(25 \%)$ in group 2.

Conclusion. The "compartment approach" reduces plate exposure rate and gastrostomy tube dependence. Revascularized osseocutaneous reconstruction is still required in dentulous patients. (C)2008 Wiley Periodicals, Inc. Head Neck 30: 709717,2008

Keywords: bone plates; mandible; mandibular neoplasms; reconstructive surgical procedures; surgical flaps

The goals of reconstruction of a lateral mandibular defect are to maintain oral competence, maintain bite force, and facilitate mastication. These goals are likely to be achieved if the mandible is brought into continuity and the oral cavity and/or the oropharyngeal soft tissues can be optimally reconstructed. While bridging, reconstruction plates are an integral part of restoring mandibu- 
lar continuity, they are alloplasts that are prone to exposure. Because of the difficulties with plate exposure, reconstructive techniques using osseocutaneous revascularized free tissue transfers evolved to reduce the incidence of this complication. ${ }^{1-3}$ In addition, the osseocutaneous tissue transfer adds enough structural integrity to the reconstructive plate to resist bite force, particularly in the dentulous patient. ${ }^{4}$ For these reasons, the osseocutaneous revascularized free tissue transfer is now considered the ideal approach to limit plate-related complications in patients with lateral mandibular defects. ${ }^{5-9}$

The problem with osseocutaneous flaps is that the associated soft tissue may not be adequate for reconstruction of complex soft tissue defects following composite extirpations of the oral cavity and oropharynx. To meet this challenge, reconstructive surgeons will reconstruct with 2 freetissue transfers to optimize both the osseous and soft tissue reconstructions. The problem associated with the use of 2 simultaneous revascularized free-tissue transfers is that both operative time and surgical complexity are increased in a patient population that can have significant comorbidities. ${ }^{10}$

At the University of Michigan, we have considerable experience with mandibular reconstruction using revascularized soft tissue and a bridging reconstructive plate. Plate exposure rates with free revascularized soft tissue reconstructions combined with bridging plates needed to be evaluated in our patient population to justify this approach and to determine which subgroups would benefit the most from this type of reconstruction. An alternative to the 2-flap approach was considered desirable for optimization of the functional result and minimization of the platerelated complications. As part of an overall change in reconstructive philosophy, we shifted our reconstructive approach to include consideration of the volume of the spaces we were reconstructing. We elected to "over-reconstruct" the volume of the resected mandible at the same time as addressing the volume loss of the other soft tissue components extirpated in the composite resection. This approach consisted of a single soft tissue flap with a bridging reconstructive plate that would possibly address plate exposure rate while optimizing the soft tissue reconstruction.

On the basis of this hypothesis, a model of "compartment reconstruction" has been developed. This approach specifically addresses the compartments that have been resected, over- replaces the volume of these compartments, and attempts to restore the contour and shape of the resected soft tissue within the oral cavity and oropharynx. Our working theory is that the increased volume of the revascularized tissue transfer keeps the external facial skin from contracting through the plate in an attempt to obliterate dead space medial to the plate. As the surgical wound contracts, the tissue lateral to the plate is "stretched over the plate," and is subjected to pressures that likely exceed the critical capillary closing pressure. This medial traction results in gradual necrosis of the tissue overlying the plate. The current study analyzes whether the "compartment approach" to lateral mandibular reconstruction can significantly reduce the plate complication rate and be an effective alternative to osseocutaneous reconstruction. In addition, it attempts to determine which clinical-demographic factors are important in choosing this reconstructive approach.

\section{MATERIALS AND METHODS}

Study Design. This retrospective case series included 40 consecutive patients with major composite defects of the lateral mandible reconstructed by surgeons in the microvascular program of the Department of Otolaryngology-Head and Neck Surgery at The University of Michigan Health System from August 1995 to March 2001.

Patient Population. Patients were included in this study if the surgical defect resulting from cancer excision included a complex lateral mandibular defect involving the entire alveolar canal, and the reconstruction was performed with a Leibinger titanium hollow screw reconstruction plate (THORP) and revascularized soft tissue flap. The indication for soft tissue free flap and bridging plate reconstruction was determined by the patient's health status as well as the nature of the soft tissue defect in patients undergoing lateral segmental mandibulectomy. The soft tissue defects were high volume such that if an osseous mandibular reconstruction had been performed, a second soft tissue revascularized tissue transfer or a multi-paddled scapula flap would be required to adequately restore the soft tissue defect. The patients were divided into 2 groups based on the reconstructive approach. Group 1 (conventional approach) included 16 patients, all reconstructed prior to September 1998, who underwent recon- 

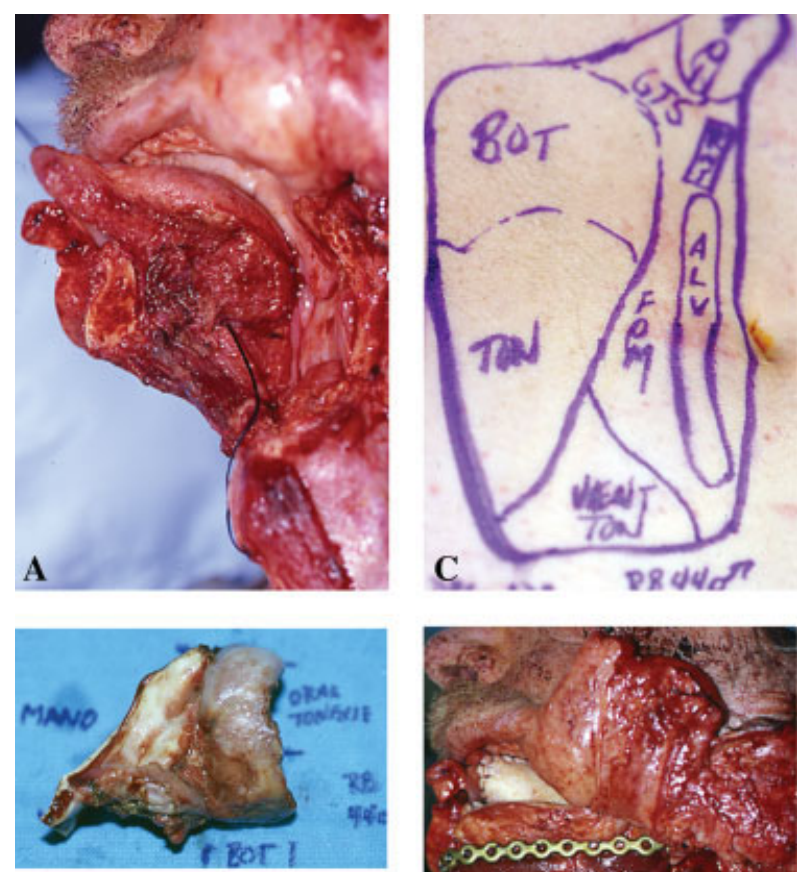

B

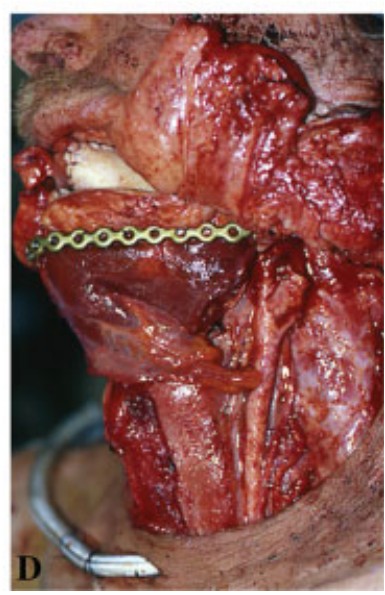

FIGURE 1. A 44-year-old man with recurrent T4 N2B squamous cell carcinoma of the base of the tongue was treated with the compartment approach reconstruction. The patient had received concomitant chemo and radiation the previous year and was continuing to smoke and consume alcohol. (A) Defect. To resect this deeply invasive squamous cell carcinoma, a hemiglossectomy, radical tonsillectomy, and mandibulectomy were performed. The resection was extended to include the medial pterygoid. The tumor also spread down the hyoglossus and involved the hyoid bone, which was included with the specimen. The cut edge of the hyoid can be seen. (B) Composite resection specimen. Includes ramus and body of the mandible, floor of mouth, lateral tongue, hemi-base of tongue, tonsil, lateral soft palate, medial pterygoid, middle constrictor, digastric, mylohyoid, geniohyoid, hyoglossus, and the hyoid bone. Note the poor quality of the oral mucosa. (C) Rectus revascularized transfer template. The rectus was chosen for its volume. The resected subunits are marked out for the mucosal reconstruction. The oral tongue, mandibular compartment, parapharyngeal compartment, and oral floor compartment required reconstruction. The template was marked out on the right rectus in the peri-umbilical zone. Rectus fascia was harvested to resuspend the larynx and support the floor of mouth reconstruction. (D) Example of compartment approach reconstruction. Note that the rectus revascularized transfer was chosen because it had adequate bulk to reconstruct the resected compartments and push from medial to lateral against the reconstructive plate. A palate tube was created between the lateral aspect of the soft palate and the cut edge of the posterior oropharynx. The rectus fascia was used to re-suspend the larynx to the reconstruction plate, which also helped to confine the reconstruction and ensure that the fat from the rectus remained against the medial aspect of the plate. struction that restored the volume of the soft tissue defect. Group 2 (compartment approach) included 24 patients, all reconstructed after September 1998, who underwent reconstruction with the compartment approach, which over-corrected the soft tissue defect around the reconstruction plate (Figure 1).

As shown in Table 1, there were 26 males and 14 females who met the inclusion criteria, with a mean age of 60 years (range, 18-80 years). The minimum follow-up was 5 years. On the basis of clinical staging, 5 patients had T1/T2 disease and 35 patients had T3/T4. Thirty-seven patients had squamous cell carcinoma as the tumor type, and 1 patient had mucoepidermoid carcinoma, mucosal

\begin{tabular}{|c|c|c|}
\hline Characteristics & $\begin{array}{c}\text { Conventional } \\
\text { approach } \\
(n=16)\end{array}$ & $\begin{array}{c}\text { Compartment } \\
\text { approach } \\
(n=24)\end{array}$ \\
\hline Mean age, y & 59 & 61 \\
\hline Preoperative BMI & 23.2 & 23.6 \\
\hline \multicolumn{3}{|l|}{ Sex } \\
\hline Male & $10(62)$ & $16(67)$ \\
\hline Female & $06(38)$ & $08(33)$ \\
\hline \multicolumn{3}{|l|}{ T classification } \\
\hline 1 & $0(0)$ & $0(0)$ \\
\hline 2 & $0(0)$ & $05(21)$ \\
\hline 3 & $06(38)$ & $05(21)$ \\
\hline 4 & 09 (56) & $14(58)$ \\
\hline Other & $01(06)$ & $0(0)$ \\
\hline \multicolumn{3}{|l|}{ Disease process } \\
\hline First primary & $09(56)$ & $10(41.5)$ \\
\hline First primary recurrence & $05(31)$ & $10(41.5)$ \\
\hline Second primary & $02(13)$ & $04(17)$ \\
\hline \multicolumn{3}{|l|}{ Neck dissection } \\
\hline None & $02(13)$ & $01(04)$ \\
\hline SND & $10(62)$ & $13(54)$ \\
\hline MRND & 03 (19) & $06(25)$ \\
\hline RND & $01(06)$ & $04(17)$ \\
\hline \multicolumn{3}{|l|}{ Flap site } \\
\hline Radial forearm & $13(81)$ & $14(58)$ \\
\hline Lateral arm & $0(0)$ & $04(17)$ \\
\hline Rectus & 03 (19) & $06(25)$ \\
\hline \multicolumn{3}{|l|}{ Defect type } \\
\hline Oral cavity and partial tongue & $06(38)$ & $04(17)$ \\
\hline Oropharynx no tongue & $07(43)$ & $15(62)$ \\
\hline Near total tongue with larynx & 03 (19) & $05(21)$ \\
\hline \multicolumn{3}{|l|}{ Radiation } \\
\hline None & $01(06)$ & $03(12)$ \\
\hline Preoperative & $06(38)$ & $09(38)$ \\
\hline Postoperative & $09(56)$ & $12(50)$ \\
\hline \multicolumn{3}{|l|}{ Dentition } \\
\hline Edentulous & $04(25)$ & $13(54)$ \\
\hline Dentulous & $11(69)$ & $11(46)$ \\
\hline Osseointegrated implants & $01(06)$ & $0(0)$ \\
\hline
\end{tabular}

Abbreviations: BMI, body mass index; SND, selective neck dissection; MRND, modified radical neck dissection; RND, radical neck dissection. Note: Values represent number of patients (\%), except as otherwise stated. 
melanoma, and osteosarcoma as the tumor type. Nineteen patients were treated for their first primary; the remaining 21 patients had either recurrent or second primary carcinomas. The radial forearm was the donor site in 27 patients, the lateral arm was used in 4 patients, and a rectus free flap was used in 9 patients. Thirty-seven patients underwent neck dissection. Twentythree patients underwent selective neck dissections, 9 underwent modified radical, and 5 underwent radical neck dissections. Three additional patients who had previously undergone neck dissections, and were currently being treated for recurrence, had neck explorations for vascular access. A total of 36 of 40 patients underwent radiation therapy. Fifteen patients received preoperative radiotherapy, 5 of whom received concomitant chemoradiation therapy. The "preoperative radiation" group consisted of patients who underwent salvage surgery after a recurrence rather than planned postradiation surgery. A total of 21 patients received postoperative radiotherapy, of which 1 patient received concomitant chemoradiation therapy. Four patients did not require radiation.

This patient population had a high mortality rate as most patients had advanced or recurrent disease. Only 6 of $40(15 \%)$ patients are presently alive and free of disease. One $(2.5 \%)$ patient is alive with disease, $22(55 \%)$ patients are dead of disease, $1(2.5 \%)$ patient died from a distant primary tumor, $9(22.5 \%)$ patients died of intercurrent illness, and $1(2.5 \%)$ patient died of unknown cause.

Approach. All surgical procedures were carried out after obtaining appropriate informed consent. Our general approach to complex lateral mandible defects is to perform an anatomic reconstruction. The Leibinger Locking System (Stryker Leibinger, Kalamazoo, MI) THORP plate was used to bridge the segmental lateral mandibular defect in all patients. (At present this implant system is no longer available. The authors are currently using the KLS-Martin threadlock system). The plates were applied to the buccal cortex of the remaining native mandible using standard techniques. A microvascular soft tissue free flap reconstruction was used to replace the soft tissue in both patient groups. This created plate coverage and maintained the mandible in its anatomic position with proper occlusion. For the "compartment approach," the revascularized free-tissue transfer was designed with a large de-epithelialized adipose component. Upon inset, the de-epithelialized portion of the flap was rolled into the parapharyngeal and mandibular compartment. The volume of the involved compartments was over-filled by approximately $50 \%$. The surface area of the defect was closed in a template based fashion, restoring the native contours of the aerodigestive tract. Once the de-epithelialized soft tissue was positioned medial to the reconstructive plate, it was sutured in position. The de-epithelialized portion was sutured to prevent it from falling out of position into the neck. Sutures were placed in the lateral aspect of the defect between the plate and the de-epithelialized portion of the flap. The deepithelialized portion was also sutured to the deep muscle adjacent to the medial aspect of the defect. Five to 8 loose, simple, 4.0 monofilament, resorbable sutures were used. Next, the lateral facial and mental SMAS (superficial muscle aponeurotic system) were sutured to the plate in a similar fashion to prevent ptosis of the overlying skin. If any suspensory muscles had been cut or the tongue base had been resected, the hyoid was also resuspended to the plate with a 2.0 simple gortex suture. Perhaps the most important point to note is that, for the compartment reconstruction to be effective, the soft tissue from the revascularized flap would have to push laterally against the medial aspect of the reconstructive plate and overfill the involved compartments (Figure 1).

Outcome Measures. Outcome measures included plate exposure, plate fracture, and oral intake ability. The time to plate fracture and exposure was recorded. Oral intake ability was classified as follows: nothing by mouth; tube feeds with oral trial; combined oral and tube feeds; nutritional supplements by mouth; oral intake with nutritional supplements; and oral intake alone. The explanatory variable was the type of reconstructive approach, either the conventional or compartment approach. The independent variables used in this analysis included the reconstructive approach, age, sex, body mass index (BMI), defect, radiation (preoperative, postoperative, or none), and dental status (presence of teeth or dentures, absence of teeth, or dentures). The defect was coded to include each involved site.

Statistical Analysis. Univariate data were tabulated for all dependent and independent variables. Bivariate comparisons between treatment groups were assessed by chi-square statistics, Fisher's exact test, Wilcoxon rank-sum test, and Kruskal-Wallis statistics. Age, sex, preoperative 
BMI, defect type, radiation status, dentition status, $\mathrm{T}$ classification, $\mathrm{N}$ classification, disease process, neck dissection type, and flap site were comparable between patients receiving either reconstructive approach. Because of the small sample size, oral intake ability was grouped into those not requiring nutritional supplements of any type and those who did require oral supplements. The logrank statistic was used for the comparison of homogeneity of "time to plate fracture" between the reconstructive groups, and between edentulous and dentulous patients.

Multivariate analysis was used to assess the factors associated with outcomes of interest. Logistic regression was used for binary outcomes including plate exposure and plate fracture. The proportional odds model was used for oral intake ability. Covariates of interest included age, sex, standardized preoperative BMI, defect type, radiation status, and dentition status. The final models were chosen through backward deletion of the covariates.

All data were maintained in a Filemaker Pro 7.0 relational database (Claris Corp., Santa Clara, California) and kept on a server designed to protect patient confidentiality at the University of Michigan. The data were exported as comma delimited text to SAS version 8.2 (SAS Institute, Cary, North Carolina). A 2-tailed $p$ value of .05 or less was considered statistically significant. This was an institutional review board-approved study.

\section{RESULTS}

Overall, 8 of $40(20 \%)$ patients experienced plate exposure, at a mean time of 10 months (range,

Table 2. Outcome measures by reconstruction.

\begin{tabular}{lcc}
\hline & \multicolumn{2}{c}{ No. of patients (\%) } \\
\cline { 2 - 3 } & $\begin{array}{c}\text { Conventional } \\
\text { approach } \\
(n=16)\end{array}$ & $\begin{array}{c}\text { Compartment } \\
\text { approach } \\
(n=24)\end{array}$ \\
\hline Plate exposure & $6(38)$ & $2(08)$ \\
$\begin{array}{l}\text { Plate fracture } \\
\text { Diet }\end{array}$ & $3(19)$ & $4(17)$ \\
Nothing by mouth & $6(38)$ & $6(25)$ \\
Tube feeds, trial oral & $1(06)$ & $3(13)$ \\
Combined oral and & $0(0)$ & $1(04)$ \\
$\quad$ tube feeds & $0(0)$ & $1(04)$ \\
$\quad \begin{array}{l}\text { Nutritional supplements } \\
\text { by mouth }\end{array}$ & $5(31)$ & $7(29)$ \\
$\quad \begin{array}{l}\text { Oral intake with } \\
\text { nutritional supplements }\end{array}$ & $4(25)$ & $6(25)$ \\
\hline Oral intake alone & &
\end{tabular}

Reconstruction Plate for Lateral Mandibular Defects

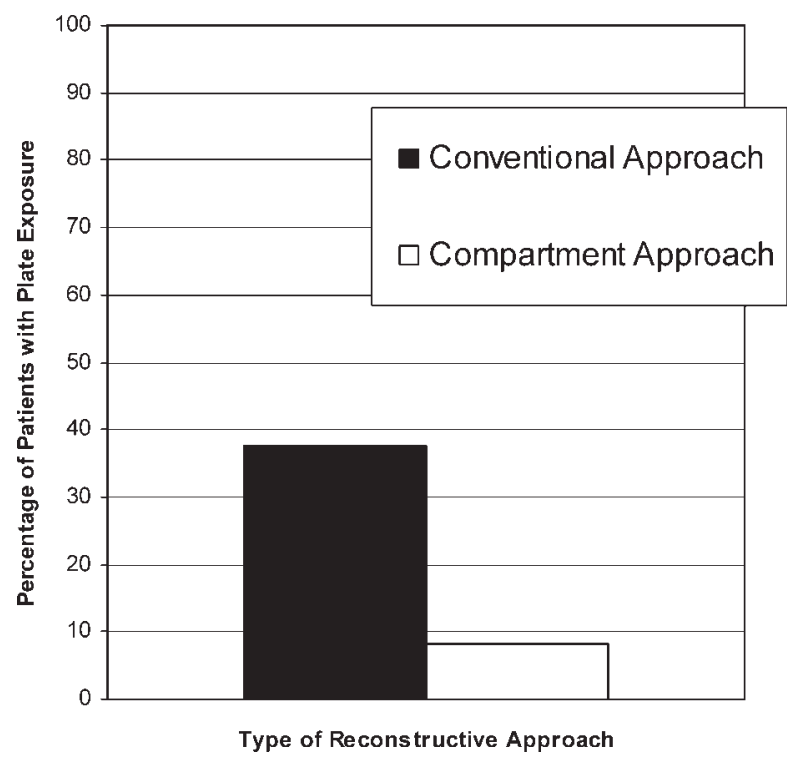

FIGURE 2. Rate of plate exposure based on reconstructive approach. Plate exposure rates were significantly lower in patients who underwent reconstruction with the compartment approach $(8 \%)$ versus the conventional approach $(38 \%)(p=$ .042).

4.7-17 months). The plate exposure rate in reconstruction group 1 (conventional approach) was 6 of $16(38 \%)$ (Table 2$)$. The plate exposure rate for group 2 (compartment approach) was 2 of 24 (8\%). This difference in plate exposure rate was statistically significant $(p=.042)$, which suggests that the compartment approach is effective in reducing the rate of plate exposure (Figure 2). By multivariate analysis, reconstructive approach was found to be the only factor associated with plate exposure $(p=.04)$. Radiation status, preoperational BMI, defect type, age, and sex were not found to be significantly associated with plate exposure. All the exposures were external.

The overall plate fracture rate was 7 of 40 $(18 \%)$, with a mean time to fracture of 21 months (range, 2.3-44 months). One of the 17 patients who were edentulous experienced plate fracture $(5.9 \%)$, whereas 6 of 23 patients who were either dentulous (22/23) or had osseointegrated implants $(1 / 23)$ experienced plate fracture $(26.1 \%)$ (Figure 3). Although this difference was clinically apparent, it was not statistically significant $(p=.20)$. However, patients who were either dentulous or had osseointegrated implants had significantly shorter $(p=.049)$ median time to plate fracture (43.9 months) than patients who were edentulous (median time not yet reached). Although radiation status was not significantly associated with plate fracture, 1 of $15(6.7 \%)$ of the patients who were 
radiated and required surgical salvage experienced plate fracture, whereas 6 of $21(28.6 \%)$ of the patients who received postoperative radiation experienced plate fracture. This higher rate of plate fracture in patients radiated postoperatively is likely related to the better diet scores as these patients consumed more complex diets than the salvage surgery patients and, as a result, would be likely to create larger plate loads. No association was found between plate fracture and covariates of interest, including reconstructive approach, radiation status, preoperative BMI, defect type, age, and sex.

Oral intake ability did show some statistically significant differences. Multivariate analysis showed that radiation status and preoperative BMI were 2 significant factors that affected oral intake ability (Table 3). Those who had combined radiation treatment after surgery had better oral intake than those who underwent radiation and subsequently required salvage surgery, with an odds ratio of $8.1(1.9,34.5)$. This represented a statistically significant difference $(p=.02)$. The odds ratio for the preoperative BMI was 1.2 (1.0, 1.4 ), which means that a 1-unit increase in preoperative BMI was associated with a $20 \%$ increase

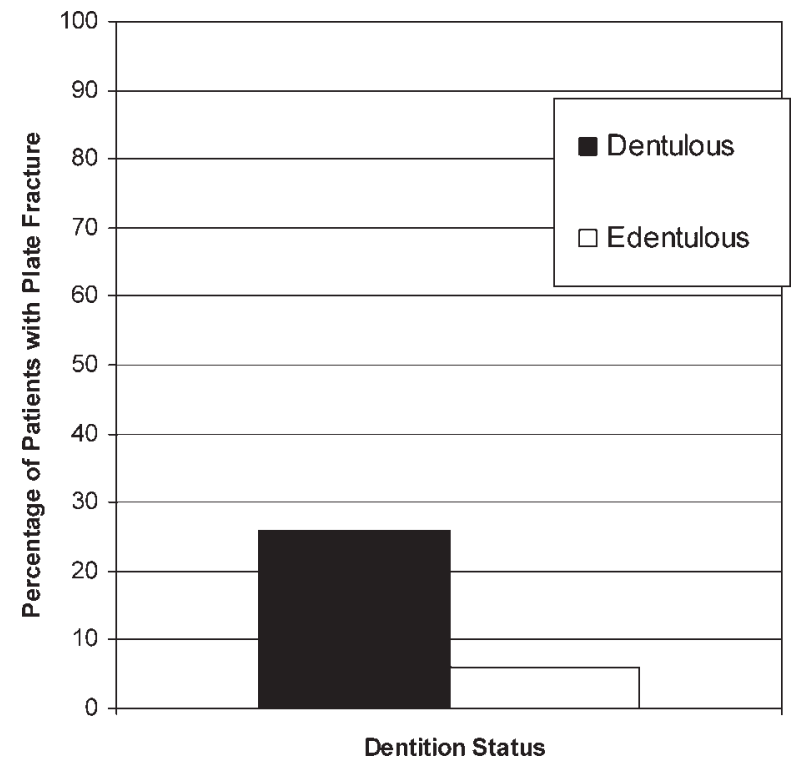

FIGURE 3. Rate of plate fracture based on dentition status. Plate fracture rates were higher in patients who were dentulous or had osseointegrated implants $(26.1 \%)$ than in edentulous patients $(5.9 \%)$. Although this difference was clinically apparent, it was not statistically significant $(p=.20)$. Median time to plate fracture, however, was significantly shorter in patients who were dentulous or had osseointegrated implants (43.9 months) than in edentulous patients (median time not yet reached) ( $p=$ .049).
Table 3. Odds ratios of variables associated with improved oral intake ability.

\begin{tabular}{cc}
\hline & $\begin{array}{c}\text { Odds ratio of improved } \\
\text { oral intake ability }\end{array}$ \\
\hline Each 1 point increase & $1.2(1.0,1.4)$, higher \\
in preoperative BMI & intake for each 1 point \\
increase in BMl & ingroved oral \\
Postoperative versus & odds of improved oral \\
preoperative radiation & intake ability if radiated \\
& postoperatively \\
\hline
\end{tabular}

Abbreviation: BMI, body mass index.

in the predicted odds for better oral intake ability in postoperatively radiated patients.

The overall rate of gastrostomy tube (g-tube) dependence was 12 of 40 (30\%) (Table 2). In group 1 , six of $16(38 \%)$ patients were strictly g-tube dependent for all of their nutrition, versus 6 of 24 $(25 \%)$ in group 2. Despite the differences in frequency, which indicated that volume reconstruction patients were less likely to require a g-tube, this did not represent a statistically significant difference. The overall high rate of g-tube dependence in this study cohort is believed to be due to the extensive nature of the reconstruction defects and the high proportion of patients under going salvage surgery (preoperative radiation).

\section{DISCUSSION}

In this study, we set out to determine if the "compartment approach" to lateral oromandibular reconstruction can reduce plate complication rates and improve oral intake ability when compared with the conventional soft tissue free flap reconstruction. In addition, we examined whether other clinical factors are important in choosing this reconstructive approach. The plate exposure rate was significantly lower in patients who underwent reconstruction with the compartment approach as compared to those with the conventional approach. This suggests that this approach is an effective alternative to osseocutaneous reconstruction with respect to the plate exposure rate. The median time to fracture was significantly shorter in patients with intact dentition versus those who were edentulous. This suggests that if a patient has teeth and significant loading is expected postoperatively, then an osseous reconstruction of the mandible may be more appropriate. In terms of oral intake following 
reconstruction, radiation status and preoperative BMI were the only significant contributing factors. Patients who received postoperative radiation had better oral intake than those who were surgically salvaged after a failure of radiation therapy (preoperative radiation). In addition, higher preoperative BMI was associated with better oral intake ability. Maintaining swallowing function after surgical salvage of previously radiated patients, particularly those with a low BMI, is difficult. In this analysis, BMI is an interesting measure that may also be a surrogate measure of the overall physical health of the patient.

"Compartment reconstruction" is 1 of many approaches to lateral mandibular reconstruction in the head and neck cancer patient that have been reported. Surgical approaches are varied and range from studies that have described using reconstructive plates with primary closure,${ }^{11}$ to reconstructive plates with soft tissue coverage, ${ }^{12,13}$ and to reconstructive plates with osteocutaneous free flaps. ${ }^{4}$ Zwetyenga et $\mathrm{al}^{11}$ described using a titanium bridging plate with no soft tissue coverage on a group of 38 patients. Although the mean operating time was only 2 hours and 30 minutes, 10 of 38 patients required plate removal at a mean time of 20.4 months. This approach has not gained favor because of the high complication rate. In addition, this approach could not be used in our cohort as the defects were too large for primary closure. Several studies have also described the use of different types of soft tissue flaps. Comparisons have been made between myocutaneous pedicled flaps and fasciocutaneous revascularized tissue transfers. There is evidence that revascularized tissue transfer decreases complication rates when compared with pectoralis flaps, although tissue transplantation did add operative time. ${ }^{13}$ The most controversy surrounds the decision to use an osseocutaneous revascularized tissue transfer versus a revascularized soft tissue transfer in conjunction with a reconstructive plate. Urken et $\mathrm{al}^{4}$ demonstrated that the plate exposure rate could be reduced to less than $10 \%$ with an osseocutaneous revascularized tissue transfer. Shpitzer et al $^{8}$ corroborated these results in a collaborative retrospective study between the University of Toronto and the Free Hospital in Amsterdam. Although complication rates were well-controlled with osseocutaneous flaps, difficulties remained with the soft tissue associated with the osseocutaneous tissue transfers because this tissue had less flexibility for reconstruction of complex oral and oropharyngeal defects. In 2003,
Blackwell published a retrospective study of 210 patients that demonstrated no significant difference in the overall reconstructive failure rates between patients with lateral mandibular defects reconstructed with osseocutaneous reconstruction versus a plate and free flap reconstruction. ${ }^{14}$ This study was observational in nature and did not specifically evaluate etiologic factors. Our report builds on this work by presenting the compartment model as an approach that specifically reduces plate exposure rates in patients who underwent reconstruction with a bridging plate and revascularized free-tissue transfer.

In our study, we used a quasi-experimental design to assess the effectiveness of the compartment approach to soft tissue revascularized tissue transfer and a bridging reconstructive plate. We studied a patient population with large, complex composite, high-volume defects, and lateral mandibulectomy, which included the entire alveolar canal. In all patients, the same type of reconstructive plate was used, and $92 \%$ of patients underwent radiation therapy either preoperatively or postoperatively. This patient group was chosen to test outcomes in patients who typically would require a complex multipaddled or multiflap reconstruction. In the historic control group, the incidence of plate exposure was similar to previous studies that assessed plate exposure rates with soft tissue and a bridging reconstructive plate. ${ }^{10}$ The significantly decreased exposure rates in our "compartment reconstruction" group are comparable to the complication rates reported in patients receiving osseocutaneous reconstruction. ${ }^{4,8,14}$ In assessing the validity of the compartment reconstruction approach, it is interesting to look at the subset of patients who had an oropharyngeal defect. Of the 6 patients who developed plate exposure in group 1, four of these patients underwent an oropharyngeal reconstruction. This patient group experienced the highest exposure rate. This is also the group in which there are multiple compartments to reconstruct, including the parapharyngeal space and mandibular space as well as the soft tissue structures in the oropharynx and lateral oral cavity. If the reconstructive plan did not address each of the involved compartments, the total volume, and the surface area of the defect, we believe that the patient would be at higher risk for plate exposure. There are not enough events to allow meaningful statistical analysis, but this finding is consistent with our overall hypothesis.

Although "compartment reconstruction" reduced the incidence of plate exposure, it did not 
have an effect on plate fracture. Our results show that a reconstruction utilizing the titanium bar with a fasciocutaneous free flap is not appropriate for patients with teeth. The bridging plate does not have the structural integrity to withstand the longterm physical stress of mastication, as demonstrated by the significantly reduced time to fracture in the dentulous group. It is likely that all patients with intact dentition reconstructed with a plate and soft tissue reconstruction will eventually experience plate fracture if they live long enough.

The type of quasi-experimental design used for this study merits a brief discussion as a potential weakness. When a historic intervention is compared to a more recent, updated intervention, the tendency is toward the updated intervention showing better results. If the same 2 interventions are evaluated with a randomized 2 group design, there is a possibility that they may no longer be significantly different. This is a recognized design flaw in historical control, quasi-experimental studies such as this study. Historical bias explains this effect, for which there are many causes. In this study, the possible causes of historical bias could relate to increasing adeptness with lateral mandibular reconstruction, improvement of patient care in the perioperative period, improvement in radiation technique, and improvement in general supportive care. All these sources of bias could be affecting the outcome in this study. The practical challenge is to find a better study design. Randomization in this patient population would be difficult, primarily because of its small size. In defense of the historical control design that was chosen, the historical group immediately preceded the group with the modified intervention. Minimization of the chronological gap between the control and modified intervention group is a well-recognized approach to minimize historical bias.

The main functional outcome measure in this study was oral intake ability. We found that the main determining factors affecting the patients' ability to maintain an oral diet were preoperative radiation and BMI. Patients who received preoperative radiation had significantly higher g-tube dependence rates than patients who received postoperative radiation. In terms of BMI, we found that a 1 unit increase in preoperative BMI was associated with a $20 \%$ increase in predicted odds for better oral intake ability. The effects of these 2 variables were significant regardless of the reconstructive approach or the patients' dentition status. We did observe that the oral intake ability was better in the "compartment approach" group, but this was not statistically significant. The observed difference between the 2 groups was large, and if the samples were larger, it is possible that more than 2 variables would be significant.

We believe that the improvement in plate exposure rate was due to specific attention to the resected compartments and anatomic subsites. Our working theory, which might explain the success of the compartment approach, is that the increased volume of the revascularized tissue transfer keeps the external facial skin from contracting through the plate in an attempt to obliterate dead space medial to the plate. As the surgical wound contracts, the tissue lateral to the plate is "stretched over the plate" and is subjected to pressures that likely exceed the critical capillary closing pressure. This medial traction results in gradual necrosis of the tissue overlying the plate. Adequate reconstruction of the volume medial to the plate (which is the preference of the authors) or placement of the vascularized tissue lateral to the plate will help counteract the medial traction. Previous reports have shown that cross-sectional reconstructive plate contour is an important factor. ${ }^{15} \mathrm{It}$ is reasonable to propose that plate geometry with a higher profile and a sharp contour could accelerate the process of tissue necrosis if critical capillary closing pressure is the important pathophysiological event. Traditional osseocutaneous revascularized tissue transfers may have been more effective than prior attempts at low-volume soft tissue reconstructions because the boney tissue medial to the plate is well vascularized, which keeps the overlying skin from contracting through the plate. Bone also has the structural integrity to resist wound contraction within the reconstructed oral cavity/oropharynx and should protect the overlying facial skin. Since the study period, the anterolateral thigh flap is more commonly used for oral cavity reconstruction. The anterolateral thigh is a bulkier alternative to the radial forearm free-tissue transfer. The bulk of this revascularized transfer should be beneficial in reducing plate complications when bone is not used.

The "compartment approach" has been effective in reducing complications related to plate exposure. Edentulous patients with large complex defects benefit from this approach. A reconstructive bridging plate in dentate patients who are expected to have good oral function will fracture without an osseocutaneous reconstruction. $\mathrm{Pa}$ tients who undergo planned postoperative radiation therapy and have a preoperative BMI $>23$ 
have much better swallowing function posttreatment. Patients who undergo surgical salvage after radiation therapy have much poorer swallowing outcomes regardless of reconstructive approach.

\section{REFERENCES}

1. Wei FC, Celik N, Yang WG, Chen IH, Chang YM, Chen HC. Complications after reconstruction by plate and soft tissue free flap in composite mandibular defects and secondary salvage reconstruction with osteocutaneous flap. Plast Reconstr Surg 2003;112:37-42.

2. Arden RL, Rachel JD, Marks SC, Dang K. Volumelength impact of lateral jaw resections on complication rates. Arch Otolaryngol Head Neck Surg 1999;125:6872.

3. Blackwell KE, Buchbinder D, Urken ML. Lateral mandibular reconstruction using soft tissue free flaps and plates. Arch Otolaryngol Head Neck Surg 1996;122:672678.

4. Urken ML, Buchbinder D, Costantino PD, et al. Oromandibular reconstruction using microvascular composite flaps: report of 210 cases. Arch Otolaryngol Head Neck Surg 1998;124:46-55.

5. Anthony JP, Foster RD, Kaplan MJ, Singer MI, Pogrel MA. Fibular free flap reconstruction of the "true" lateral mandibular defect. Ann Plast Surg 1997;38:137-146.

6. Takushima A, Harii K, Asato H, Nakatsuka T, Kimata Y. Mandibular reconstruction using microvascular free flaps: a statistical analysis of 178 cases [see comment]. Plast Reconstr Surg 2001;108:1555-1563.
7. Thoma A, Khadaroo R, Grigenas O, et al. Oromandibular reconstruction with the radial-forearm osteocutaneous flap: experience with 60 consecutive cases [see comment]. Plast Reconstr Surg 1999;104:368-378; discussion 379-380.

8. Shpitzer T, Gullane PJ, Neligan PC, et al. The free vascularized flap and the flap plate options: comparative results of reconstruction of lateral mandibular defects. Laryngoscope 2000;110:2056-2060.

9. Deschler DG, Hayden RE. The optimum method for reconstruction of complex lateral oromandibular-cutaneous defects. Head Neck 2000;22:674-679.

10. Boyd JB, Mulholland RS, Davidson J, et al. The free flap and plate in oromandibular reconstruction: long-term review and indications. Plast Reconstr Surg 1995;95: 1018-1028.

11. Zwetyenga N, Pinsolle J, Siberchicot F, MajoufreLefebvre C. Reconstruction of lateral mandibular defects with dynamic bridging plates. Br J Oral Maxillofac Surg 2002;40:307-312.

12. Kiyokawa K, Tai Y, Inoue Y, et al. Reliable, minimally invasive oromandibular reconstruction using metal plate rolled with pectoralis major myocutaneous flap. J Craniofac Surg 2001;12:326-336.

13. Cordeiro PG, Hidalgo DA. Soft tissue coverage of mandibular reconstruction plates. Head Neck 1994;16:112-115.

14. Head C, Alam D, Sercarz JA, et al. Microvascular flap reconstruction of the mandible: a comparison of bone grafts and bridging plates for restoration of mandibular continuity. Otolaryngol Head Neck Surg 2003;129:48-54.

15. Blackwell KE, Lacombe V. The bridging lateral mandibular reconstruction plate revisited. Arch Otolaryngol Head Neck Surg 1999;125:988-993. 